\title{
BIOCORROSION DES ACIERS EN EAU DE MER NATURELLE, Conditions d'utilisation
}

\author{
L. Lemoine, J. Guezennec \\ Ifremer
}

La corrosion des aciers en eau de mer naturelle a fait l'objet de nombreuses études qui ont montré l'influence des propriétés physico-chimiques de l'eau de mer (salinité, $\mathrm{O}_{2}$ dissous, $\mathrm{pH}, \mathrm{T}^{0}$, vitesse des courants, ...) sur les modes et vitesses de corrosion. Les micro et macrosalissures ont également une influence sur les mécanismes de corrosion en modifiant directement ou indirectement par les produits de leur métabolisme, les phénomènes de corrosion.

Pour identifier le rôle de chaque paramètre, il est nécessaire de pouvoir disposer de résultats de mesures électrochimiques, d'analyses de produits de corrosion, d'examen de surface et également d'observations bactériologiques et biochimiques. Les études de corrosion marine actuellement réalisées associent de manière plus courante ces moyens d'analyses pour avoir accès à ce type de données et tenter de préciser le rôle des micro-organismes, notamment sur les phénomènes de corrosion.

Pour l'acier en eau de mer, le cas des bactéries sulfato-réductrices a été depuis longtemps mis en évidence pour la corrosion localisée. Pour les aciers inoxydables, la fixation d'un voile biologique qui s'établit dès l'immersion de tout substrat en eau de mer naturelle a pour effet d'élever le potentiel d'électrode jusqu'au potentiel de piqûre de l'acier et provoquer l'attaque de ce dernier.

Toutefois, il est rare que les aciers soient utilisés sans protection cathodique (ouvrages portuaires, structures offshore, pipes,...) et que l'eau de mer de circuits (faisceaux tubulaires d'échangeurs en acier inoxydable, ...) ne soit pas traitée par chloration ou autres procédés. Il est donc nécessaire de tenir compte des conditions d'utilisation des matériaux, celles-ci modifiant les paramètres d'environnement propre à initier des phénomènes de corrosion.

Les résultats présentés ci-après ont pour but de montrer différentes approches utilisées pour identifier le rôle de micro-organismes dans différents cas d'utilisation des aciers en milieu marin. Les essais sont réalisés en eau de mer naturelle où les micro-organismes viennent se fixer sur les substrats. 


\section{Etude du comportement d'aciers au carbone et faiblement alliés en eau de mer. Effet du voile biologique}

\section{Mesure de perte en poids et profondeur de piqûre}

Des essais de tenue à la corrosion marine d'aciers faiblement alliés ont été entrepris dans différentes conditions d'exposition in situ sur la station d'essais de l'Ifremer à Brest et en laboratoire, en eau de mer filtrée. Les aciers contenaient différentes teneurs en chrome, aluminium, nickel, molybdène, afin de diminuer le taux de corrosion pour une utilisation sans protection cathodique. Les essais in situ ont été effectués sur des échantillons de $300 \times 360 \times 5 \mathrm{~mm}$ de dimensions. Après 6 mois d'exposition les éprouvettes sont recouvertes d'une couche épaisse de salissures. En parallèle les essais effectués en eau de mer filtrée $(600 \mu \mathrm{m})$ en laboratoire sur petites éprouvettes n'avaient pas la même couche de macrosalissures. Les résultats obtenus sur les pertes en poids et profondeurs de piqûres pour les mêmes durées d'exposition ont montré une assez bonne similitude dans le classement des tenues respectives des alliages, avec toutefois des différences notables de morphologie d'attaque et de profondeur de piqûre (fig. 1); ceci avait été attribué, en l'absence de résultats d'analyse précis, à la présence de micro-organismes pouvant se développer sous les couches épaisses de salissures marines et modifier ainsi les faciès d'attaque.
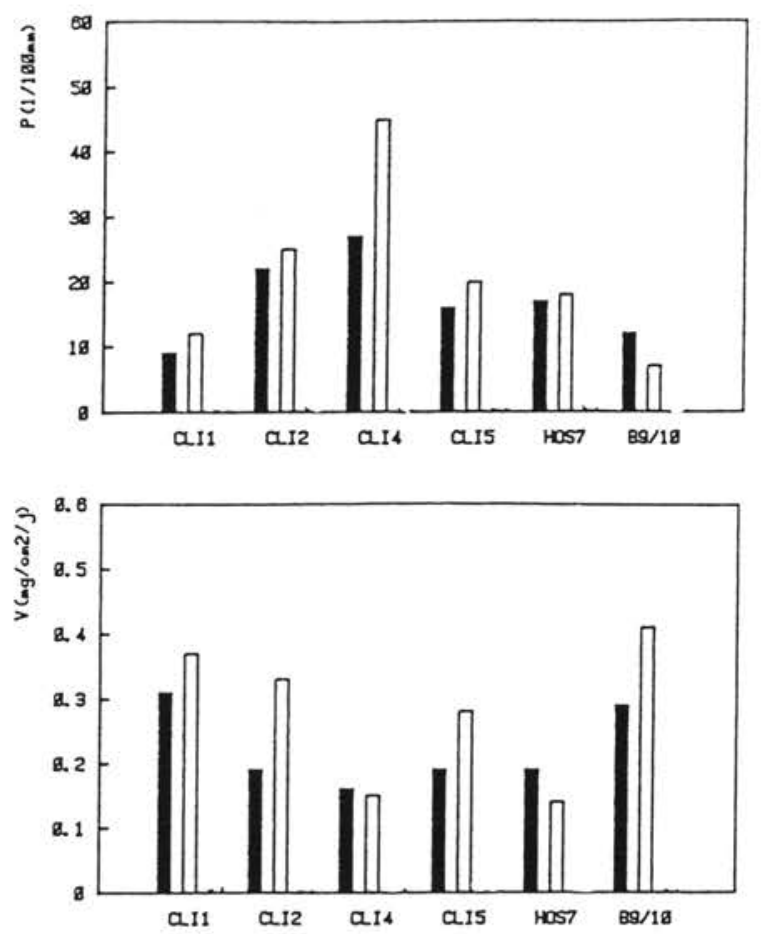

Fig. 1 - Perte en poids et profondeur de piqûre sur acier faiblement allié. 16 mois in situ

1 6 mois en laboratoire

\section{Étude par impédance}

Les études réalisées "in situ» ont été complétées dans un deuxième temps par des mesures électrochimiques sur les mêmes aciers immergés en eau de mer naturelle.

Des diagrammes ont été tracés au potentiel de corrosion pour diverses durées tout au long du vieillissement des éprouvettes. La forme de ceux-ci, notamment ceux relevant des nuances alliées, implique qu'ils contiennent plusieurs boucles; résistance de transfert (Rt) et résistance de polarisation $(\mathrm{Rp})$ n'ont donc pas dans notre cas la même signification. Ceci peut être corrélé avec l'absence de droites de Tafel sur les courbes intensité potentiel [1].

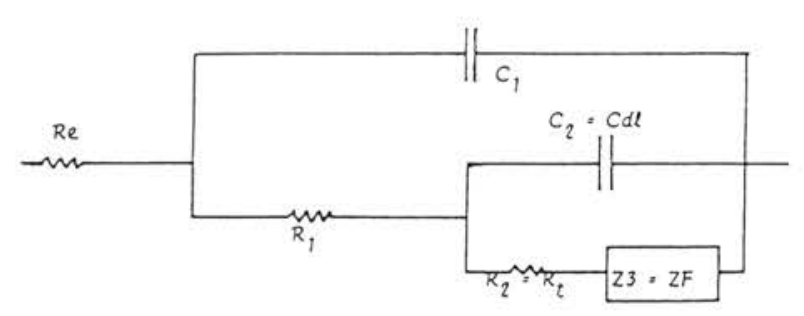

Fig. 2 - Schéma électrique équivalent pour l'analyse des diagrammes d'impédance de l'acier dans l'eau de mer.

A partir des résultats des ajustements effectués ainsi que des interprétations données par différents auteurs, il est possible de proposer le schéma équivalent représenté sur la figure 2 .

$R_{1}$ et $C_{1}$ peuvent être attribués à l'influence d'un dépôt à la surface de l'électrode. La mesure du produit $\mathrm{R}_{2} \mathrm{I}$ à plusieurs potentiels sur des échantillons rouillés en cellules à circulation durant 4 mois donne les valeurs assez proches de celles attendues pour le produit Rt. I (de 120 à $160 \mathrm{mV}$ pour la partie cathodique et de 20 à $40 \mathrm{mV}$ pour la partie anodique). Ceci justifie donc le choix de la seconde boucle comme celle représentant la capacité de double couche en parallèle sur la résistance de transfert.

Les valeurs de la capacité de double couche sont généralement très grandes (de l'ordre du $\mathrm{mF} \mathrm{cm}{ }^{-2}$ ). Celle-ci augmente de façon quasi linéaire avec le temps dans le cas de la nuance non alliée et de façon moins importante pour les autres. Ces différences traduisent très certainement des modifications des couches de rouille par les éléments d'alliage.

Il faut signaler que la forme de la seconde boucle capacitive, notamment dans le cas de l'acier au carbone, peut s'éloigner notablement d'un cercle dans sa partie haute fréquence. Ce phénomène a déjà été signalé comme pouvant provenir de l'influence d'une couche de magnétite poreuse et conductrice.

Bien que la vitesse de circulation de l'eau ait une influence importante sur la vitesse de corrosion de nos aciers, il n'a pas été possible de mettre en évidence la présence d'une impédance de diffusion. L'impédance faradique $\left(\mathrm{Z}_{3}\right.$ ou $\left.\mathrm{F}_{\mathrm{F}}\right)$, obtenue par suppression des deux premières boucles se ramène, après 6 mois environ, à une simple résistance pour les aciers 1 et 2 , tandis qu'une boucle capacitive peut généralement être obtenue pour l'acier 3 [3].

Malgré les tentatives faites pour identifier les différents mécanismes de corrosion, il n'a pas été possible de mettre en évidence clairement l'effet des micro-organismes et les phénomènes associés à la présence de l'impédance $\mathrm{Z}_{\mathrm{F}}$.

\section{Identification du rôle des bactéries}

L'activité propre des micro-organismes composant le voile biologique, la complexité du biofilm présent sur les surfaces placées dans un environnement naturel oblige à ne pas considérer uniquement et spécifiquement un métabolisme, mais également à considérer les effets de symbiose et synergie pouvant exister à l'intérieur du biofilm.

Une approche expérimentale a été tentée à l'aide d'une cellule à 2 compartiments séparés par une membrane poreuse. Un premier compartiment est rempli d'un milieu d'étude inoculé avec des bactéries. Le second est rempli avec le même milieu, mais sans bactérie (fig. 4). Les produits du métabolisme des micro-organismes peuvent traverser la membrane poreuse, mais pas les bactéries; ainsi, il est possible de séparer le rôle des bactéries de celui des produits de leur métabolisme.

Ce type d'essai a été entrepris avec de l'eau de mer filtrée dans laquelle sont ajoutés des éléments nutritifs. Plusieurs expérimentations ont été réalisées en inoculant le milieu avec des bactéries vibrio-natriegens (mode aérobie), ainsi qu'avec des bactéries sulfatoréductrices. Des diagrammes d'impédance ont été tracés à différents temps d'exposition avec apport continu d'éléments nutritifs et après arrêt. 


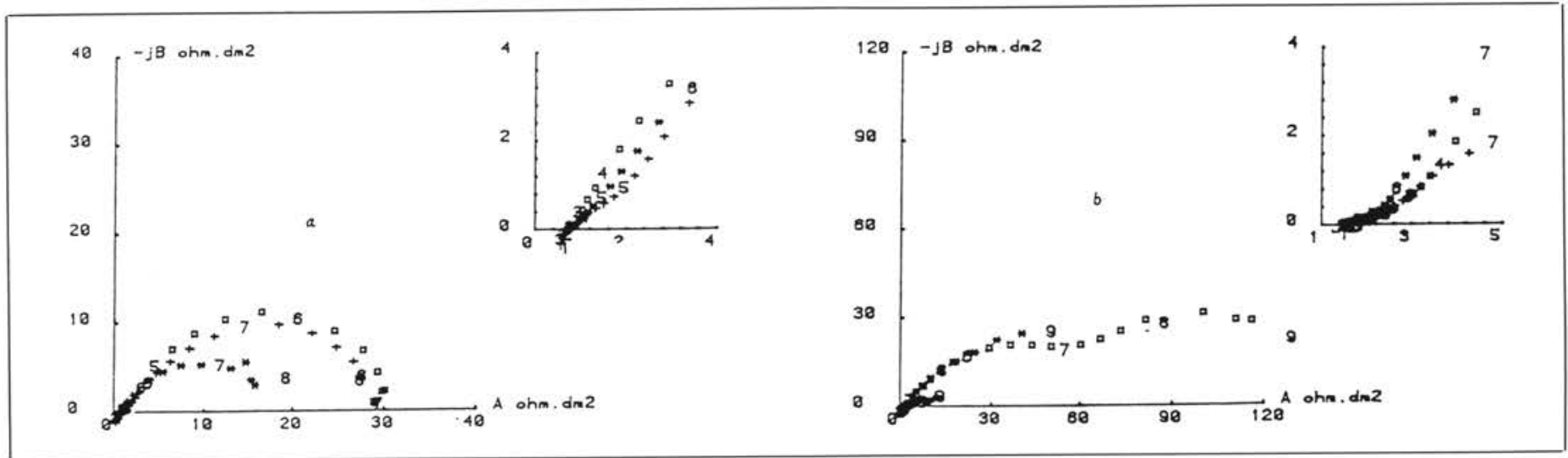

Fig. 3 - Diagrammes d'impédance électrochimique au potentiel de corrosion; a : 3 mois en immersion; b : 18 mois en immersion (+ : nuance 1; $:$ nuance 2, $\square$ : nuance 3).
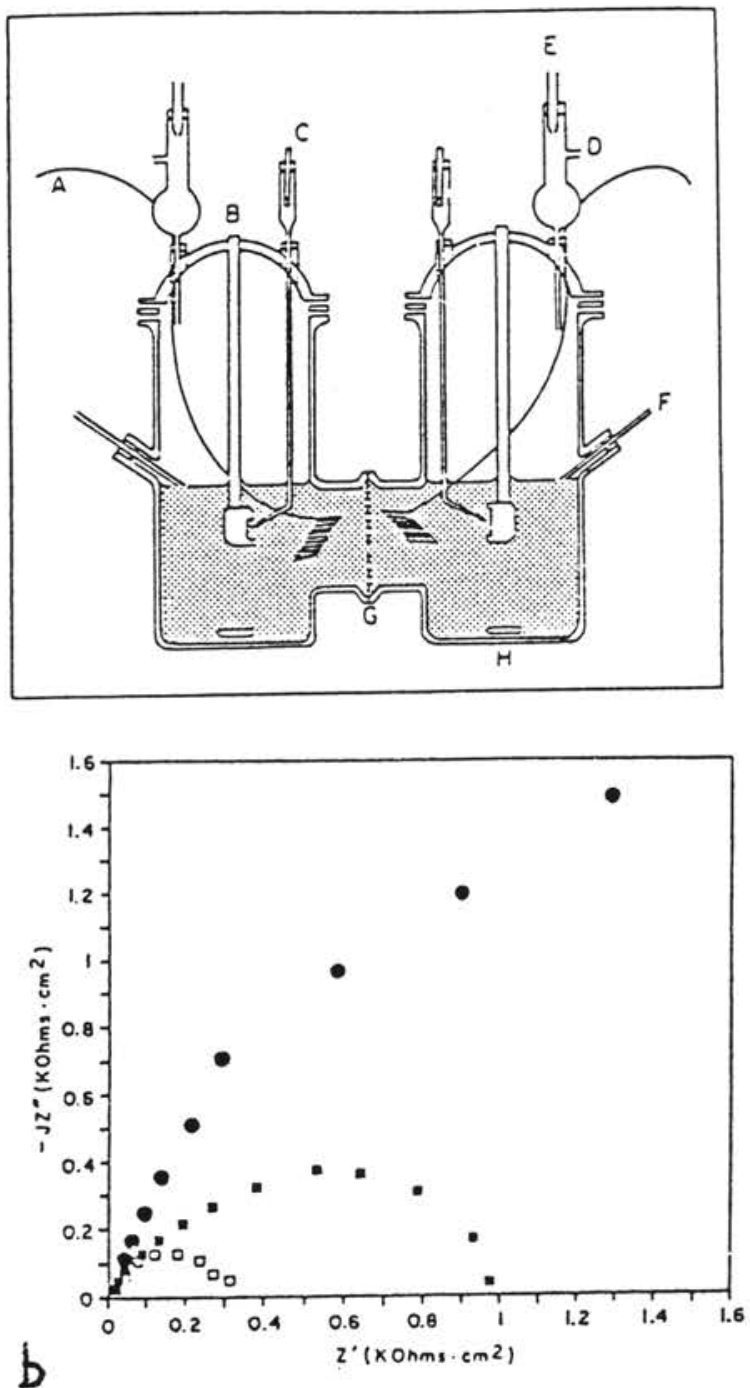

Les résultats présentés dans la figure $4(\mathrm{~b}, \mathrm{c}, \mathrm{d})$ montrent que les bactéries natriegens ont pour effet de réduire la valeur de la résistance de transfert et donc d'accélérer la corrosion. L'inoculation de bactéries SBR dans le milieu ne montre pas une accélération sensible de la corrosion lors d'un apport continu d'éléments nutritifs. Par contre, l'arrêt de cet apport a pour effet d'accélérer la corrosion par

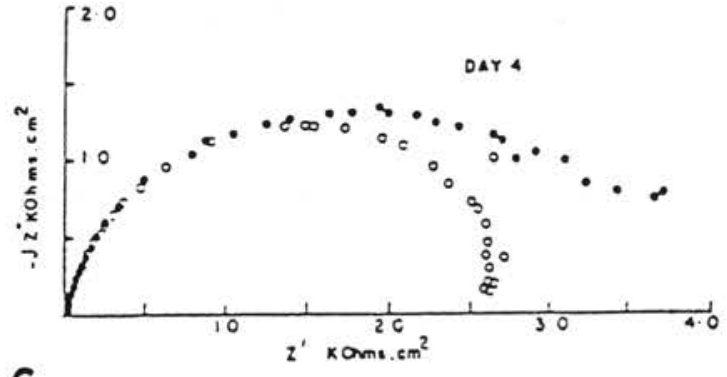

C

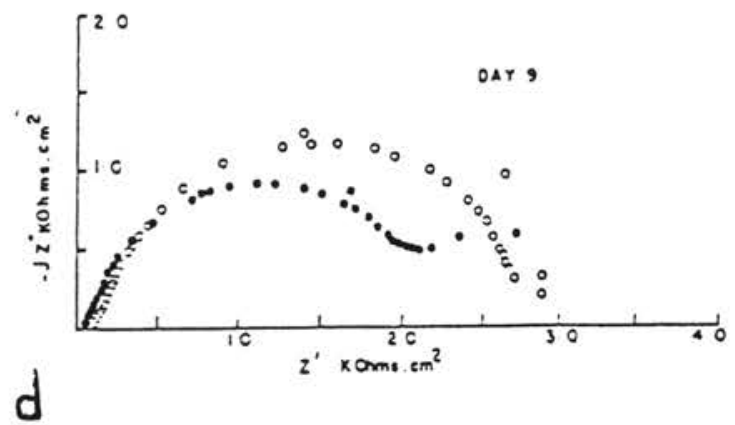

Fig. 4

(1) diagramme to

b $\{$ diagramme après 3 jours sans vibrio natriegens

$\square$ diagramme avec vibrio natriegens

(o vibrio natriegens seule

c 0 vibrio natriegens + SRB

d avec renouvellement éléments nutritifs (4 jours) après arrêt ( 9 jours). 


\section{Effet du voile biologique sur le comportement d'aciers inoxydables}

\section{Cas des matériaux d'échangeurs de chaleur des centrales énergie thermique des mers}

Pour les centrales Énergie Thermique des Mers, une efficacité maximale est demandée aux échangeurs de chaleur pour un bas coût de revient, afin d'accroître la rentabilité de telles installations [3].

Les recherches effectuées aux États-Unis ont montré l'importance du voile biologique qui encrasse les échangeurs, spécialement dans le cas d'un cycle fermé et la nécessité de maintenir un bon coefficient d'échange thermique par des méthodes de nettoyage chimique et mécanique. Une épaisseur de voile de $100 \mu \mathrm{m}$ abaisserait de $30 \%$ le rendement d'une centrale.

Pour la fabrication des échangeurs de chaleur en eau de mer, les alliages de titane sont souvent employés pour leur excellente résistance. Cependant leur coût de revient est élevé et leur approvisionnement peut être problématique. D'autres alliages sont utilisables comme les aciers inoxydables et alliages d'aluminium, mais leur comportement dans des conditions telles que rencontrées dans ces échangeurs doit être évalué.

Des mesures de coefficient d'échange de chaleur entre l'eau de mer et la paroi d'un tube en titane ont été effectuées à l'aide d'un dispositif spécialement créé à cet effet. Les résultats obtenus mettent en évidence une réduction de $50 \%$ du coefficient d'échange de chaleur au bout de 30 jours d'exposition, correspondant à une épaisseur de voile de l'ordre de $120 \mu \mathrm{m}$ (fig. 5).

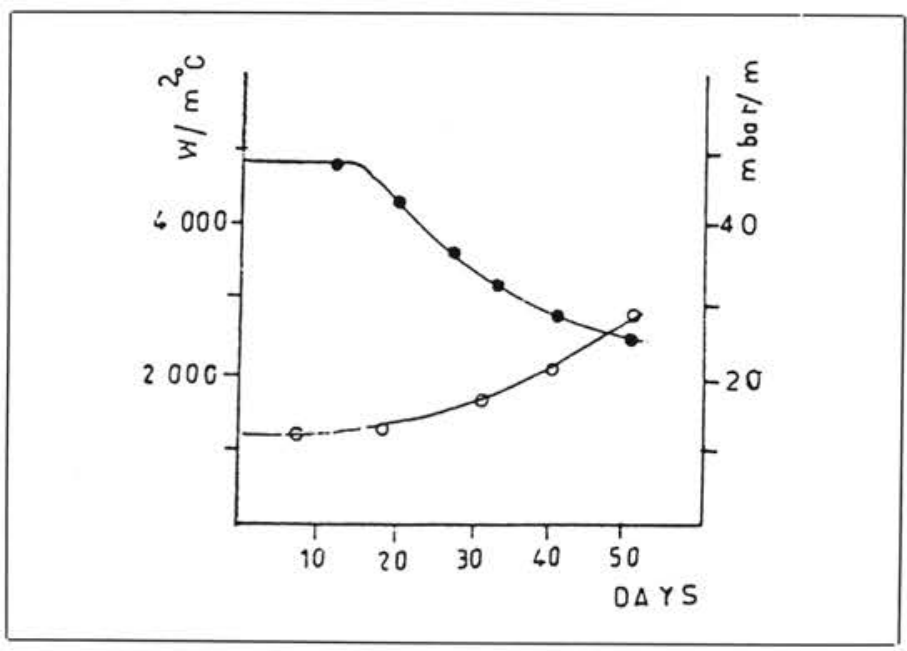

Fig. 5 - Évolution du coefficient d'échange de chaleur et de la chute de pression dans un tube de titane.

Il est donc nécessaire d'éviter la formation du voile biologique par des méthodes chimiques et mécaniques. Des essais ont été entrepris sur des cellules comprenant 7 tubes de diamètre $25 \mathrm{~mm}$, avec chloration de l'eau de mer et nettoyage en continu des tubes par nettoyage mécanique.

La chloration de l'eau de mer a pour effet d'accroître le potentiel d'électrode des aciers inoxydables. Toutefois le nettoyage mécanique a pour effet de maintenir à un niveau bas le potentiel d'électrode des aciers testés. Aucun cas de corrosion sur les aciers n'a été observé dans ces conditions d'essais sur les aciers testés.

\section{Comportement des aciers inoxydables}

La formation d'un voile biologique sur toute surface métallique exposée en milieu marin peut avoir comme conséquence principale l'initiation de corrosions à caractère localisé, liée à la seule présence et à l'activité des micro-organismes composant ce voile. Il convient cependant de distinguer l'effet du biofilm pris dans son ensemble de celui de l'activité métabolique des micro-organismes qui contribuent à la formation de voile biologique. La présence d'un voile biologique sur les aciers inoxydables a pour conséquence une remontée des potentiels d'abandon de ces aciers vers des valeurs plus nobles, proches pour certains d'entre eux du potentiel de piqûration.

Cette action du biofilm apparaît liée selon les auteurs, soit à une action enzymatique catalytique des micro-organismes sur la cinétique de réduction de l'oxygène, soit à une action catalytique des ions métalliques piégés dans le biofilm sur cette même réaction de réduction, soit encore à des modifications de paramètres physicochimiques à l'intérieur même du biofilm. Cependant, aucune de ces hypothèses suffit à elle seule à expliciter cette évolution des potentiels et qu'en fait, une conjonction de ces différents mécanismes et autres possibles, correspondent à la réalité. De nombreuses études restent encore à réaliser en ce domaine, et tout d'abord, celles conduisant à une meilleure connaissance de la nature et de la structure du voile biologique.

\section{Protection cathodique}

La protection cathodique est largement utilisée en milieu marin comme forme de protection contre la corrosion des alliages placés dans cet environnement. Une valeur de $-850 \mathrm{mV} / \mathrm{ECS}$ est généralement préconisée comme potentiel de protection des aciers doux en milieu marin aéré. En l'absence d'oxygène, ce potentiel doit être modifié, modification liée à la présence et activité de microorganismes anaérobies, telles les bactéries sulfato-réductrices, dans le milieu et sur les surfaces $[4,7]$.

En milieu naturel aéré, la mise en place d'une protection cathodique a pour conséquence principale la formation d'un dépôt calcomagnésien lié à la réduction de l'oxygène et l'alcalinisation entrâinant la précipitation de sels de calcium et de magnésium. La présence et la formation d'un voile biologique sur les surfaces peuvent influer sur la mise en place et la durabilité de cette protection.

Nous avons donc procédé à Ifremer à une série d'expérimentations ayant pour but d'étudier l'influence du voile biologique sur la protection cathodique en milieu naturel, et sur des monocultures bactériennes marines. Les résultats obtenus sur des courtes durées (1 semaine à 15 jours) d'expérimentations démontrent :

a) une faible influence de la formation du voile biologique sur la mise en place d'une protection cathodique sur de l'acier inoxydable placé en conditions naturelles, $[5,6]$.

b) les différences essentielles observées entre expérimentations conduites en milieu naturel et sur monocultures bactériennes, différences liées aux cinétiques de formation du dépôt calcomagnésien et du biofilm, variables selon les expérimentations.

En conditions anaérobies, et plus spécifiquement dans les sédiments marins ou à l'intérieur d'un biofilm, la production d'hydrogène cathodique peut avoir comme conséquence une modification de la structure de la population bactérienne et le développement de bactéries utilisant cet hydrogène comme donneur d'électrons, telles les bactéries sulfato-réductrices [9].

Durant près de 3 ans, nous avons donc procédé à des études visant à mettre en évidence la relation possible pouvant exister entre la production d'hydrogène lors de la mise en place d'une protection cathodique et la modification de structure de population bactérienne et de développement de bactéries sulfato-réductrices dans les sédiments marins. Ces études ont été faites en fonction du potentiel de protection $(-800$ à $-1100 \mathrm{mV} / \mathrm{Ag} . \mathrm{Agcl} \mathrm{Cl} / \mathrm{eau}$ de mer) à deux températures $\left(12^{\circ}\right.$ et $\left.37^{\circ} \mathrm{C}\right)$. Les principaux résultats montrent que l'hydrogène produit cathodiquement à des potentiels égaux ou inférieurs à $-1000 \mathrm{mV} / \mathrm{ECS}$ favorise la croissance de bactéries sulfato-réductrices (fig. 6) à l'interface métal-sédiment, et qu'un arrêt momentané, dans ces conditions de la protection mise en place, augmente considérablement la vitesse d'initiation de la corrosion Une augmentation de température va dans le même sens, favorisant le développement d'espèces spécifiques de bactéries sulfato-réductrices. 


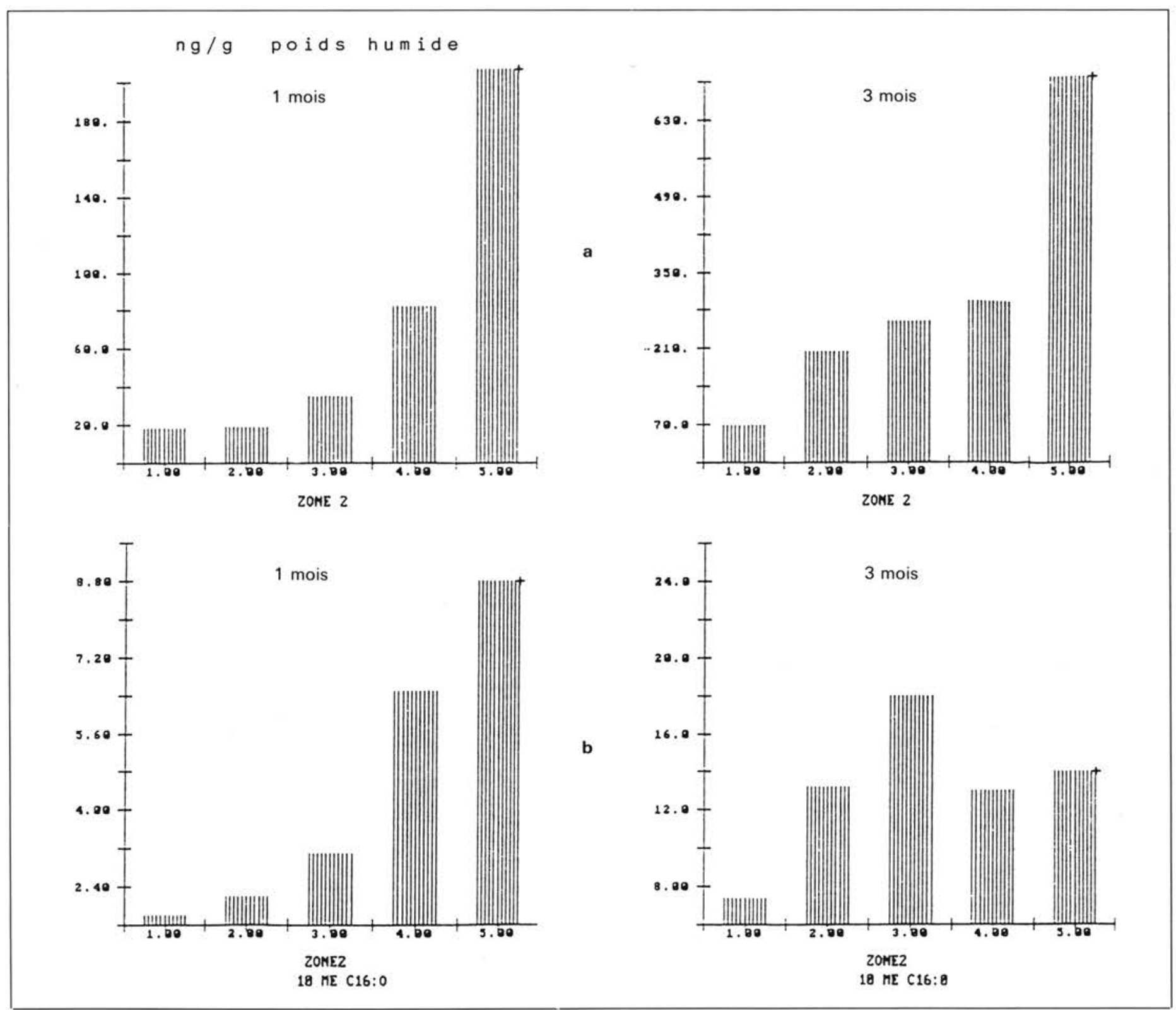

Fig. 6 - Évolution de la structure de la population bactérienne en fonction du potentiel de protection cathodique :

a) biomasse totale, b) concentration en acide gras caractéristique d'une espèce de bactérie SRB.

1 : potentiel libre; $2:-700 \mathrm{mV} / \mathrm{ECS} ; 3:-800 \mathrm{mV} / \mathrm{ECS} ; 4:-900 \mathrm{mV} / \mathrm{ECS} ; 5:-1000 \mathrm{mV} / \mathrm{ECS}$.

\section{Conclusions}

Les études réalisées en eau de mer naturelle sur le comportement d'aciers au carbone et d'aciers inoxydables montrent qu'à la complexité de la composition chimique de l'eau de mer, vient s'ajouter l'activité propre des micro-organismes. L'identification précise du rôle de ces bactéries est difficile à réaliser et les approches expérimentales ont permis de corréler des résultats de mesures électrochimiques avec des observations d'ordre biochimique ou bactériologique. Cette identification nécessite une poursuite des recherches avec d'autres techniques expérimentales pour mieux préciser les caractéristiques physico-chimiques du milieu $\left(\mathrm{pH}, \mathrm{O}_{2}\right.$ dissous, ...), sa composition (présence d'acide particulier, de polymères, ...), la nature des micro-organismes et une bonne compréhension de leur métabolisme.

Toutefois, en eau de mer, il convient que ces recherches tiennent compte de l'environnement de ces matériaux qui sont rarement utilisés sans protection supplémentaire (peinture, protection cathodique, ...) pour les aciers de construction métallique et chloration ou autres systèmes de lutte contre les salissures pour les circuits eau de mer dans lesquels sont présents des aciers inoxydables.

\section{BIBLIOGRAPHIE}

[1] $\mathrm{Ph}$. Blanchard, L. Lemoine, Étude de la corrosion d'aciers en eau de mer par mesure de l'impédance électrochimique.

[2] N. Dowling, J. Guezennec, L. Lemoine, A. Tunlind, D. C. White, 1988, Corrosion, 44 pp. 869-874.

[3] L. Lemoine, J. Guezennec, Corrosion and Biofouling of OTEC heat exchangers, Oceans 85, San Diego.

[4] M. Therene, Corrosion bactérienne dans le sédiment marin, étude de la protection cathodique. Thèse Université Paris VI, 1989.

[5] L. Fiksdal, J. GuezenneC, 1988, Int. Congress on Marine Corrosion and Biofouling, Valencia, Spain.

[6] J. GuezenNEC, E. ANTOINE, 1990 (soumis pour publication à Matériaux et Techniques).

[7] J. Guezennec, M. Therene, N. Dowling, D. C. White, Proceedings of the 7th Int. Congress on Marine Corrosion and Fouling, Valencia, Spain 1988.

[8] N. Dowling, J. Guezennec, L. Lemoine, A. Tunlind, D. C. White, 1988, Corrosion, 44 , pp. 869-874.

[9] J. L. Honegger, I. Lauriol, A. Criaud, S. Sainson, J. Guezennec, Proceedings of the workshop on Deposition of solids in Geothermal systems, Reykavik, 1988. 